Uluslararası Mühendislik

Cilt/Volume:11 Sayı/Issue:1 Ocak/January 2019

Araştırma Makalesi / Research Article

\title{
Design of a Distributed Control System with Fuzzy Logic Controller and PLC in Wireless Sensor Network Based Industrial Environments and Monitoring the System with RFID
}

\author{
Akif Durdu ${ }^{1}$ iD, Üzeyir İlbay Bozkurt ${ }^{1}$ iD, Mehmet Celalettin Ergene ${ }^{1}$ \\ ${ }^{1}$ Konya Technical University, Robotics Automation Control Laboratory (RAC-LAB), 42075, Konya
}

Başvuru/Received: 19/02/2018

Kabul/Accepted: $12 / 12 / 2018$

Son Versiyon/Final Version: 31/01/2019

\begin{abstract}
Nowadays industrial applications are built on automatic control systems. The main reason is to convert these control systems and industrial factories into smart ones. By converting them to smart factories, a high-efficiency rate can be acquired. Generally, automatic control systems are controlled by classical logic via PLC (Programmable Logic Controllers). In this method, many problems can be met with. The most important one of these is that it is complicated to create a numerical control unit. Trying to control the system with the traditional way without creating a model can lead us to complicated algorithms. At the same time, complicated algorithms can cause wrong orientations in the system. However, the fuzzy logic, which is one of the intelligent control methods, can help us to create the system just with linguistic expressions and some rules without requiring a mathematical model. In this way with smart control methods, efficiency can be obtained in factories. Besides control systems, factories can be made smart. RFID technology is the central element of this process. The factorymaterial communication is provided via RFID technology. Thus, factories can communicate with the material that is produced without any human intervention. The development of smart factories and the rapid improvements in automation systems caused demands in wireless technology to rise. As a result of these demands, wireless sensor networks became a critical subject, and its use is widespread. These systems provide us to send the data in a certain distance without any loss of the data, also removes all the cables in the workplace. In this study, a control system, distributed in a wireless sensor network based industrial places, is controlled by a traditional PLC method and fuzzy logic. At the same time, the system is monitored by RFID and a solution is proposed to a smart factory application.

Key Words
\end{abstract}




\section{INTRODUCTION}

Logic is a subject of study of reasoning methods. A reasoning method means that to create a new proposition from the present proposition. In classical logic, if a proposition is $\mathrm{p}$, it must be either true or false, so the truth-value of the proposition is 1 or 0 . For more than a century two-valued classical logic dominated the world. However, this traditional two-valued logic brings many problems along and there were failures at its truth-values. Fuzzy logic part is designated as a transition to the absolute truth and proposes a value in the range of the binary system which classical logic tells. This generalization provides the reasoning performance by giving fuzzy propositions namely uncertain results (N. Siddique, 2013).

In classical logic, variables have to be one of the values such as; cold-hot, zero-one and young-old. At the beginning of the 1920s, Polish researcher Jan Łukasiewicz came up with the idea that is the opposite of this classical logic. In fact, by telling that classical logic can have the intermediate values, he laid the foundations of many-valued logic. Max Planck in 1937 by publishing an article on Philosophy of Science journal, he has drawn the first fuzzy set curves via applying object sets to this valued logic. The first idea of fuzzy logic is put forth by Lotfi Zadeh in 1965 with his Fuzzy Sets article. However, in the Western World of the time, these ideas were approached with suspicion and got huge responses. In the last 30 years, classical logic gave its place to the logic known as "fuzzy" and started to being used in technological devices. In here verbal expressions from daily speaking language are added to the process while modeling (J. Kavulmaz, 2002).

With microelectronics, the latest developments in the area of the sensor and wireless communication technology provided a new network structure; wireless sensor devices run on batteries. The self-configured structure called wireless sensor network consists of little devices equipped by individual sensors and a wireless receiver. The primary purpose of a wireless sensor network is to collect the data from the environment and send the observed data to an analyzable reporting site. Wireless sensor devices at the same time can also answer the queries sent by a control site. Finally, under certain conditions, wireless sensor devices can be equipped by actuators for mobility. These networks are sometimes specifically called wireless sensor and actuator networks (M.A. Labrador and P.M. Wightman, 2009).

Wireless sensor networks possess many useful aspects such as low power consumption, reliability, accuracy, ease of maintenance and lower cost. This structure can contain many maintenance-free nodes. When these nodes analyzed alone, their coverage may be low; however, when they together form a network structure, this coverage may be increased. For example, a network structure formed by many nodes can be seen in Figure 1. Additionally, sensor nodes can be used in the areas where life is in danger, e.g., to observe living creatures in wild areas. In this way, they can accomplish their sensing task by working four seasons (M. Yazar, 2015).

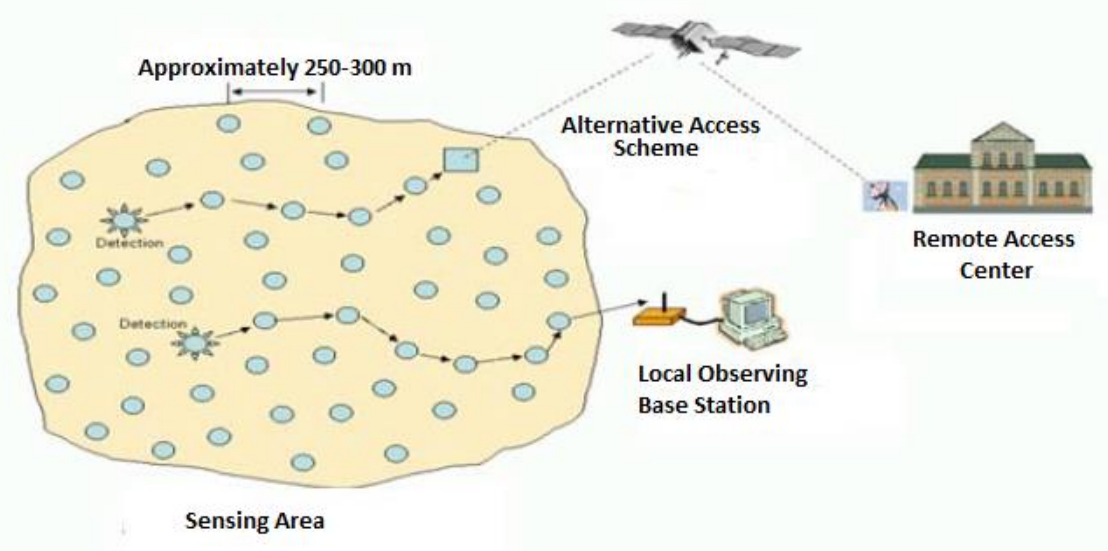

Fig. 1. Wireless sensor networks (G.H. Choi, G.S. Choi, and J.H. Jang, 2009)

Due to economic and technological reasons, nowadays power skills, memory and communication quality of wireless sensor devices are restricted. Because of that in the wireless sensor technology, efficient algorithms and protocol designing about energy and necessary calculations are focused. At the same time, because it is used at simple data focused on observing and reporting tasks, its application area is so restricted. On the other hand, wireless sensor networks, which can do more than this, are developed. More improved functions and multimedia data transfer feature is added. With new network architectures, in heterogenic devices and expected improvements in the technology, eliminates these restrictions. Thus, the application spectrum for wireless sensor networks is being developed significantly (M.A. Labrador and P.M. Wightman, 2009).

Distributed control system architectures consist of different units in the field. These units can be wireless sensor nodes, smart sensors, velocity control devices, network gateways, and computers. An example of a distributed control system architecture is shown below (Fig. 2.). The main thing about distributed control systems is that they have a network system. All of these units told about are connected to this network node and communicating each other. Control system foresees to control these units from one 
node and provides the process automation to be done from this node. There is a real-time information exchange with the control unit. However, there can be a delay appeared due to the distributed control. To make this delay the most efficient is one of the essential things in system designing (M. Anand, S. Sarkar, and S. Rajendra, 2012).

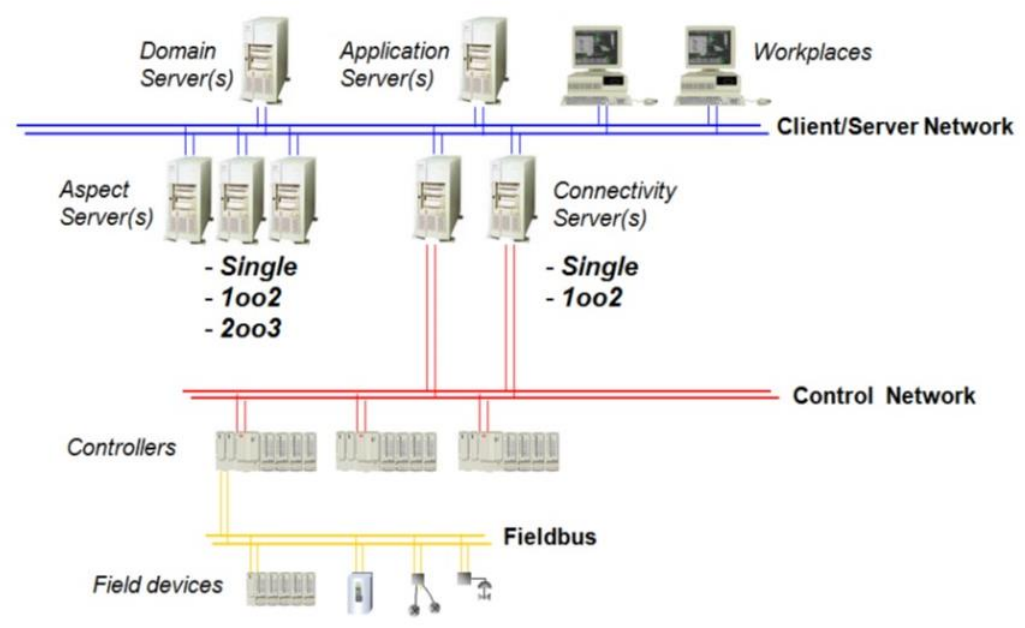

Fig. 2. Distributed control system architecture (M. Anand, S. Sarkar, and S. Rajendra, 2012)

The radiofrequency idea is introduced first time during the time of the Second World War. By transmitting and receiving back again radio waves from a certain point, radar systems are created (G. Ozbek, 2014). The development of radar systems laid the groundwork for the RFID technology. In aviation, it is used to recognize the allied and the hostile countries. The RFID technology reads and writes with radio frequency and does the identification process. Owing to the automatic identification process, the necessity of manual data input will be disappeared. Labor and at the same time the data accuracy also will be at the top level (A. Üstündağ, 2008).

It is seen that until programmable logic controllers (PLC) were developed, in automation and control systems relayed electrical control systems were being used. However, nowadays with the increase of the efficiency and the quality of the automation systems it is observed that it is improved continuously. In this improvement progress, PLC controlled systems are essential for automation systems to adapt themselves as quickly as possible. In automation systems, PLC is an automation unit with its specific numbered inputs and outputs, has a different communication typed connections, ensures the control applications are performed (S. Kurtulan, 2010).

The commercial use of PLCs started in 1969. Produced and developed by Modicon Company to use instead of relayed electrical control circuits. After the first PLCs used in industry and achieved success, big companies such as General Electric, Siemens and Schneider produced PLCs with higher quality and cost. As an alternative to these products companies such as Omron and Delta produced products (W. Bolton, 2006).

In (J. Lin and R.-J. Lian, 2010) a method to improve the performance of a plastic injection-forming machine is developed. Difficulties in producing non-linear and complicated plastic parts are mentioned. For such system, self-organized fuzzy controllers are designed. The designed system has online learning skills. It updates the automatic fuzzy rules and decides the reasonable control. For injection heavy holding pressure and injection screw velocity, PID and fuzzy logic control are applied. As a result of the fact that fuzzy logic gives better performance is shown.

In ( P. Singhala, D. Shah, and B. Patel, 2014) by using fuzzy logic, low cost improved temperature control is done. The system consists of a heater, fan and a temperature sensor. A temperature value is taken from the user via a keyboard. The temperature is kept constant on the taken temperature value by using a few rules with fuzzy logic in the system. Operation processes of fuzzy logic inputs and outputs are determined, and fuzzification and defuzzification processes are done. As a result, it is specified that the application of this technology is not difficult. It is shown that the solution, which is cheaper and faster, is produced in this way.

Wireless sensor networks in health observing applications are used in (J. Yick, B. Mukherjee, and D. Ghosal, 2008). Infant monitoring, blood pressure warning, and observing applications are done. Sleeping positions of infants are observed and provided them a safer sleep. For the infants are in wrong positions, their parents are warned.

With the help of wireless sensor networks, in (I. Morsi and L.M. El-Din, 2014), a habitat monitoring application is made. By placing more than one sensor nodes to the creatures' nests, big spots are monitored. The effect of the monitored values such as temperature and humidity to the creatures are researched. 
In (P. Chanak and I. Banerjee, 2016), a fuzzy rule base is developed in order to detect the broken sensors and reusing them in a wireless sensor system. It is told that the detection of these sensors is significant for service quality. Some of the chosen nodes are classified with fuzzy inference. Experiments are done on it by creating variable network scenarios. The experiment result is compared with the current algorithms, and the most available algorithm is proposed. It is shown that the proposed method detects only faulty sensors and provides its reuse in order to increase the network performance. It does not affect energy consumption is told. It is observed that, with the help of the proposed data orientation algorithm, the errors of the sensors are corrected.

A SCADA/PLC system is used to control petrol and gas refineries with traditionally DCS in (I. Morsi and L.M. El-Din, 2014). In this way by presenting a real system for the application, a particular application method is applied. The design is made by dividing the factory into four main parts. Various sensors are used to measure the temperature and the velocity in the system. The system is very successful and stable to reduce human power and increase life safety. In SCADA systems huge data can be recorded and monitored in anywhere around the world while the monitoring is being done. With this system, the result is obtained faster than DCS.

In (C.K.H. Lee, K.L. Choy, K.M.Y. Law, and G.T.S. Ho, 2014), smart data management sourced RAS (source allocation system) system is developed for source allocation. This system consists of human, information, and control supporting functions for production efficiency. In the system for smart deciding, fuzzy logic to analyze the data and RFID (radio frequency identification) system for verification of the result is developed. They confirmed the system by applying it to a clothing facility. At the end, it is observed that the system is more efficient with fuzzy logic.

In (A.B. Kılıç, 2015), first of all, the theoretical information about RFID is laid stress. Afterward, studies are done about a special C\# software program. A software program is developed for the roll call in a school, the entrance and the exit of the students are observed from the main desk. It is told that this technology is developing and in the future, it will take place in many applications.

A fuzzy system is proposed about a practical application of PLC system in a servo mechanic system in (I. Dumitru, N. Arghira, I. Fagarasan, and S. Iliescu, 2010). As a method fuzzy logic is used. In this servo mechanic system, velocity control application is laid stress. In the designed system, PLC and a fuzzy logic system are connected to each other.

In many factory fields, products completed on installation and production lines are carried via forklifts. These lines are generally consisting of more than one station. The main aim in here is to carry as many as products as soon as possible. If this conveyance system is observed, forklifts are carrying the products from the stations randomly. In a word, even if the factory is empty, the forklifts are still wandering around the factory, and they go to the station irrespective of any limitation when they see that the products are finished on the station. As a result of this energy and time waste occurs.

In this study, first of all, a factory environment is thought like as mentioned before. In this environment, stations in absolute numbers are designed and by creating a suitable network topology, a wireless network structure is simulated. Information in this simulation is considered as a distributed system, and a software program is developed in the CODESYS program. The algorithms created in this software put into practice. An operator panel is developed in simulation place, and how the factory will be observed and the intervention will be made is shown. RFID read-write tags are placed on the forklifts and the stations to control the production. This RFID control is observed from the operator panel, and necessary settings are made. As mentioned before, forklifts are deciding randomly which station to choose. For this process, a fuzzy model is developed on a suitable software program. With this model, appropriate input and output functions will be created in order to save energy and time. The comparison between algorithms based solution and a fuzzy model solution is made, and the performance analysis is made at the end of the study.

\section{FUZZY LOGIC}

If Figure 3 will be examined, the people between 0-30 years old are young, 30-50 years old are, and over 50 is considered old. According to these rules, when a person at the age of 29.5 is considered as young, a person at the age of 30.5 is considered as middle-aged. If it is thought for a controller, if the sets made of physical sizes are divided with such sharp lines, it is unavoidable that to see rapid changes at the control output. As opposed to the cited above fuzzy logic can make softer qualification than the sharp logic's binary concept and it by qualifying open/close, cold/hot as little open/little closed and a little cold/a little hot creates a more appropriate logic. If we handle the age topic again, as a person at the age of 33 can be considered as middle-aged, he/she can be considered as young as well. Sometimes the title of middle age sometimes young could be suitable. As it can be seen in Figure 4, fuzzy sets are convenient to create such flexible logic. The fact that sets are not divided with sharp lines provides the possibility for a 33 years old person to be considered young in some rate and middle-aged in some rate (A. Özek and M. Sinecen, 2004). 


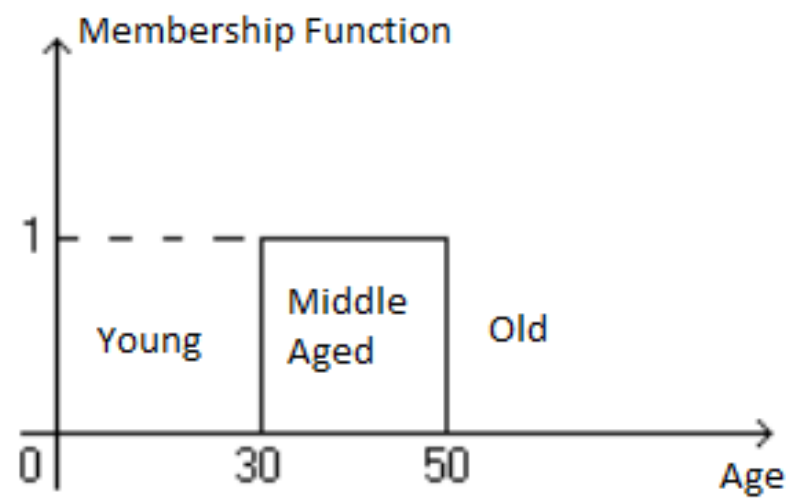

Fig. 3. Age distribution membership function belongs to the classical set (E.A. Tiryaki and R. Kazan, 2007)

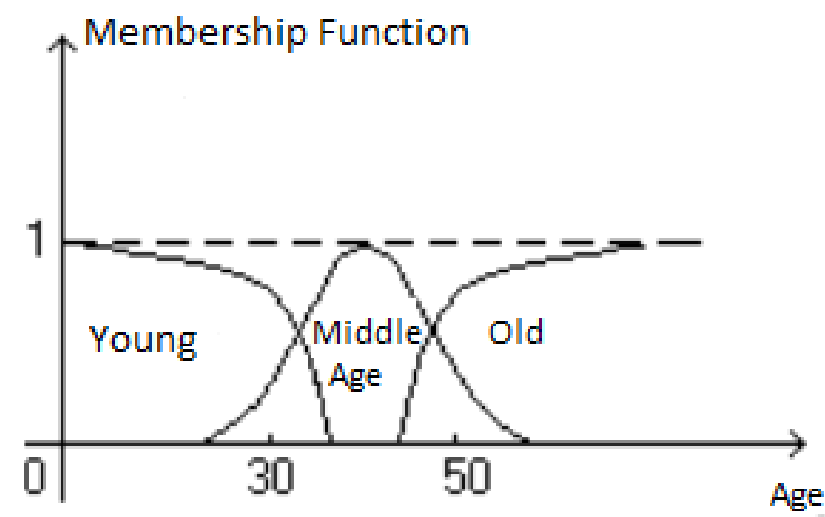

Fig. 4. Age distribution membership function belongs to the fuzzy set (E.A. Tiryaki and R. Kazan, 2007)

\subsection{Fuzzy and Classical Sets}

According to the classical set theory, there are only three members $\boldsymbol{x}_{1}, \boldsymbol{x}_{2}, \boldsymbol{x}_{\mathbf{3}}$ in the set B under the age of 40 , because of this, the border is so wide between over 40 years old and under 40 years old faculty members. In Figure 5 this border can be seen clearly. However, for the fuzzy set theory, the set B does not only contain these three members s $\boldsymbol{x}_{1}, \boldsymbol{x}_{2}, \boldsymbol{x}_{\mathbf{3}}$ but it also contains other members in changing degrees. Distribution of ten teaching staff's real age on the $\mathrm{x}$-axis is shown in Figure 5 . When this graph is examined, it can be seen that s $\boldsymbol{x}_{\mathbf{4}}$ and $\boldsymbol{x}_{\mathbf{5}}$ are not the members of the set B, but respectively with their rates of 0.7 and 0.3 membership degrees, they can be considered as partial members (Y. Bai, H.Q. Zhuang, and Z.S. Roth, 2005).

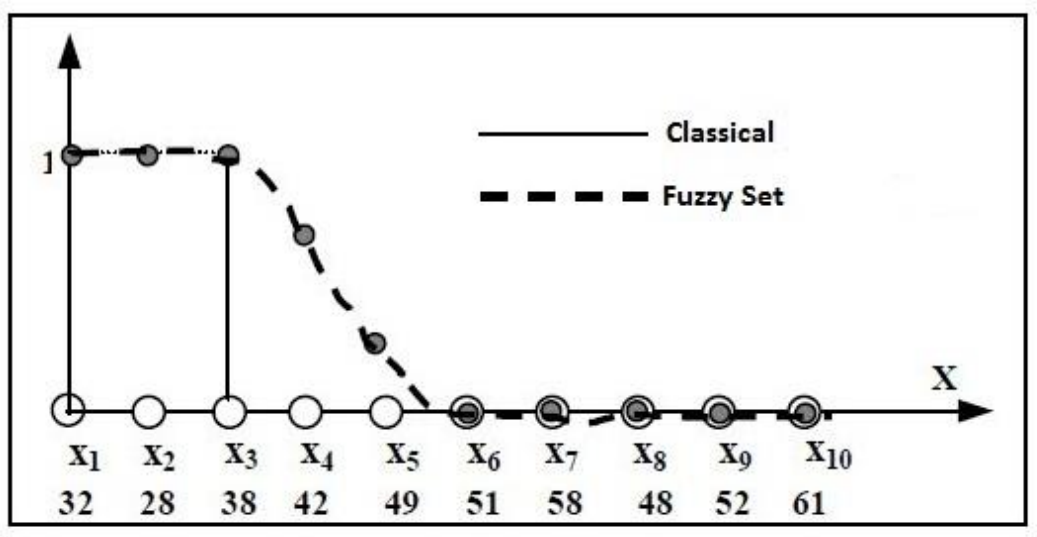

Fig. 5. The comparison between classical and fuzzy sets (Y. Bai, H.Q. Zhuang, and Z.S. Roth, 2005)

\subsection{Fuzzy Logic Control Structure}

Nowadays in automation systems, in order to obtain an excellent automatic control these systems must be known well. This is also very important to design fuzzy control. The main point of fuzzy control is creating a rule base with verbal expressions by using supervisors' knowledge and experiences. Control systems are made not by classical and fuzzy control algorithms but by the rules created by verbal expressions (R. Arınç, 2003). 
A system, which will perform the fuzzy deciding process, can be designed by gathering this given information in one place with fuzzy logic control. In Figure 6 a fuzzy logic control structure is shown. This structure can be assembled under four main titles.

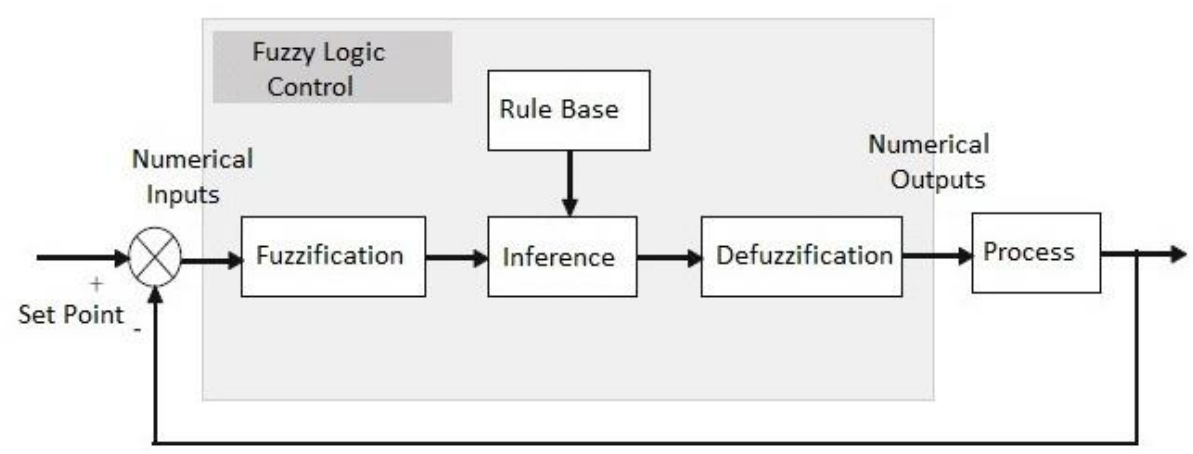

Fig. 6. Fuzzy logic control structure (N. Siddique, 2013)

1. The input data taken from the outer world and has the absolute numerical value is converted to verbal expressions via membership functions. This step is called fuzzification unit.

2. Rule base, which is the second step, creates propositions with verbal expressions and fetches the control rules.

3. Expressions obtained from the fuzzification are compared with the expressions created in the rule base, and as a result, verbal expressions are obtained again. This step is called inference.

4. If the fuzzy output of the inference step is sending information to an, e.g. system, these outputs must be converted to a language which the system can understand. This conversion process is performed in the defuzzification step (S. Y1lmaz, 2007).

\section{WIRELESS SENSOR NETWORKS AND CODESYS}

The architecture of a wireless sensor device is so simple. In Figure 7 the general structure of the sample architecture is given. A wireless sensor device consists of a processor, memory, radio transmitter, antenna, power supply, and input/output interface. These devices are equipped with low powered microcontrollers. CPUs of advanced microcontrollers consume less energy; however, wireless sensor devices maintain batteries. Because energy is significant for these devices. Setting some parts of these devices to sleep mode is also critical. Generally, in these wireless devices, there are 16-32 bit RISC (reduced instruction set computing) working with little energy. Duties of some of the units create a sensing node is mentioned below (M.A. Labrador and P.M. Wightman, 2009).

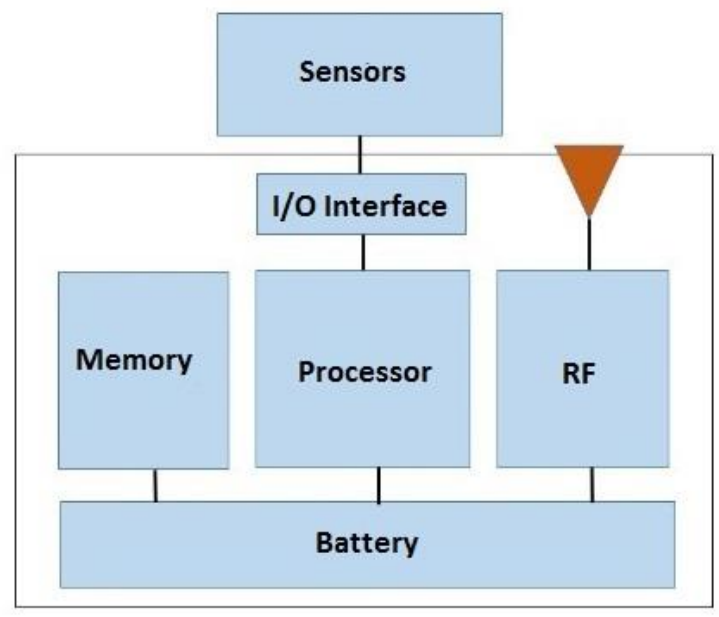

Fig. 7. A general structure of a wireless sensor device (M.A. Labrador and P.M. Wightman, 2009)

Wireless sensor network architectures were developed over time and are being improved. The most important reason for this is there are new and skilled devices. At the beginning, wireless sensor networks consisted of a plain topology that does the measurement with only one variable. These networks formed by many wireless sensor devices distributed in the area they are relevant. These devices with the network they form, they can observe even very remote regions and analyze it. New applications 
require a significant number, hundreds maybe thousands of nodes. These applications call for new architectures for the efficient transmission of the wireless sensor data (M.A. Labrador and P.M. Wightman, 2009).

In basketball, first of all, players are selected after that to form team players should come together. For wireless sensor networks, the same logic applies. Devices can be connected in a few orders, or network structure should be given to the topology. Radios show logically how to connect these topologies. Their environmental regulations, of course, can be different. There are many different network communication methods in wireless networks. Sample network topologies are given in Figure 8 (R. Faludi, 2010).

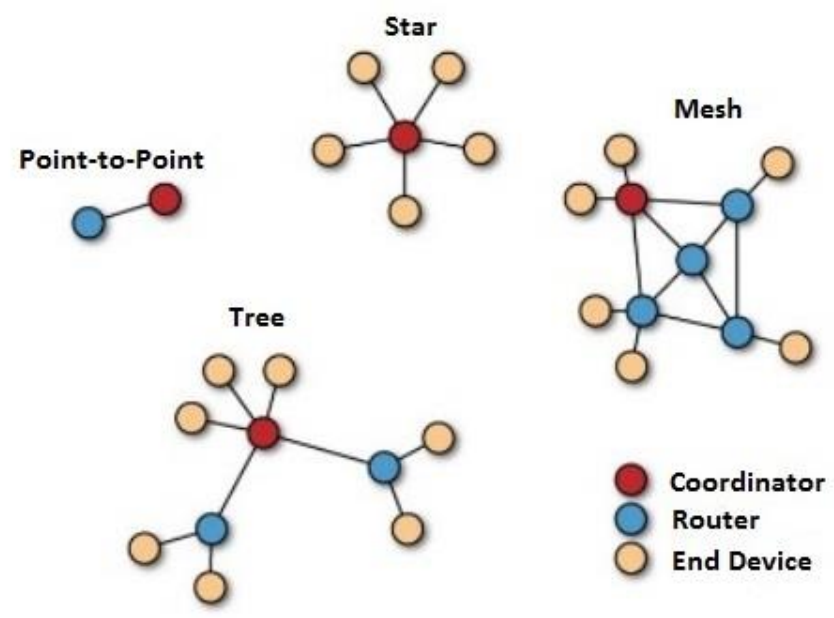

Fig. 8. Sample network topologies (R. Faludi, 2010)

\subsection{Point-to-Point Network Topology}

The topology forms the most straightforward network structure, consists of two devices, coordinator and router. At this point, the coordinator may be a gateway or a node. However, to create a network, one node should have a coordinator. In here the node can be a router or an end device. An example of a topology is shown in Figure 9.

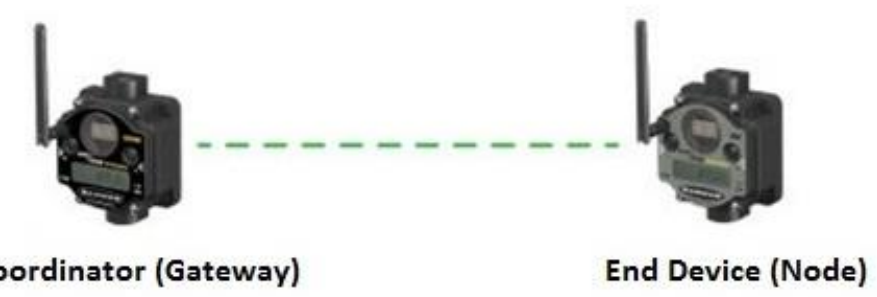

Fig. 9. Point-to-Point network topology [21].

\subsection{Star Network Topology}

It is a network, which works by transmitting and receiving data from one central station to many node control points. In this network connection method, node points can exchange data with only the central station. Every node cannot exchange data among themselves. If there will be any disconnection between the central station and any node, other nodes would not be affected by this situation. In Figure 10, an example of this network structure is shown. The most significant advantage of this topology is that the delay between the central station and the nodes is so low. For example, in an application, the delay between the central station and a note was monitored as $100 \mathrm{~ms}$. Besides the power consumption of the nodes can be kept under control. As every central station has a particular power gain, they can sense only within a certain range. In this network architecture, every node has to be within the range (T. Soylu, 2012). 


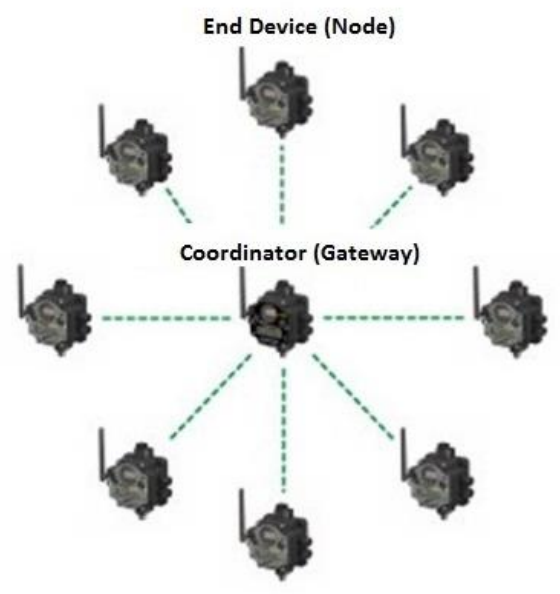

Fig. 10. Star network topology (M. Raffin, 2010)

\subsection{Tree Network Topology}

Tree network topology is a special kind of point-to-point structure. It is a way of communication between the devices in a hierarchical order. It is formed by more than one point-to-point topology. Physically in this topology, the total number of nodes in the network can be one less than the number of point-to-point connections in the network. If this network turns into less than three layers, then this network becomes a part of the star topology. This network topology consists of the coordinator, router and nodes. There is no necessity of nodes being within the range of the coordinator. Because routers, which are the members of this topology, can act as repeaters. Repeaters transmit the information taken from the nodes to the coordinator, which is a superstructure. In this way, the range is expanded. As shown in Figure 11, communication of the node number three with the coordinator is provided via a router. In the same time, the communication of the node number three with the node number five is provided via the coordinator. As the branching increases in this topology, the duties of the routers increase proportionally (F. Demirel, 2010).

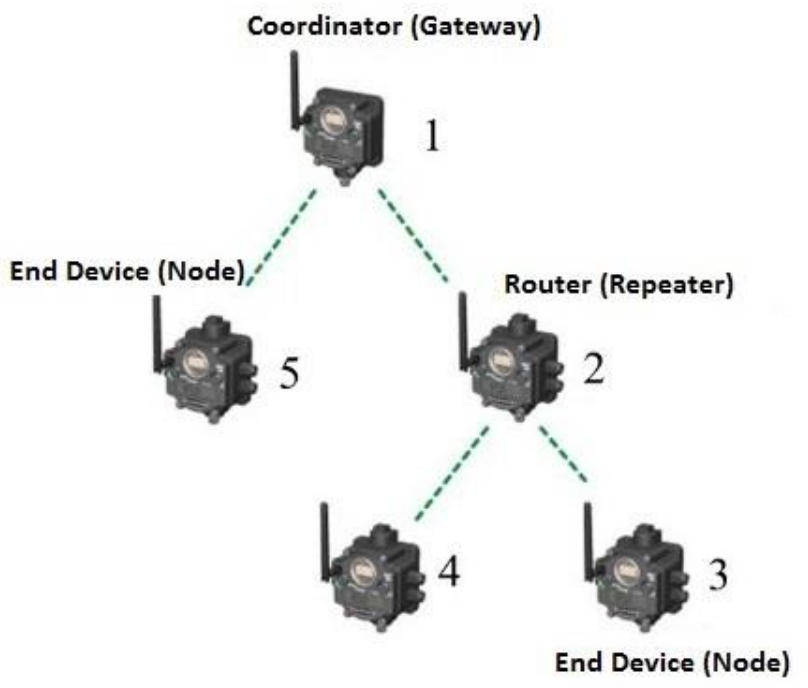

Fig. 11. Tree network topology (M. Raffin, 2010)

\subsection{Codesys Software Program}

To be able respond the rapid developments of industrial applications, it is necessary to be able to study with many different types of PLC structures and manage them. However, nowadays one if the biggest problem is being bonded to only one brand and having difficulties in different systems. Software programs in different frameworks leave the users in a difficult situation. Codesys eliminates this situation and proposes a software structure independent from the hardware. Codesys abbreviation stands for "Controller Development System". It confirms the IEC 61131-3 industrial standard. Codesys proposes a flexible programming facility to the user, and at the same time, it is open source and free. Therewithal engineers can create an application for their needs. A program written for a brand of PLC via Codesys can be adapted to another brand of PLC with just some small hardware changes. Regarding programming language it offers a widened structure. In this way, the program can be simplified by using languages peculiar to the application. It is possible to add different types of POU (Program Organization Unit). There are three varieties such as function, function block and standard program. 


\subsection{DCS and Master-Slave Structure}

If we consider the infrastructure of distributed control systems, they consist of master and slave members. Slave units can be a gateway, wireless sensor, velocity control device or a proportional valve. Their duties are only to collect information and transmit to the superstructure master member. Slave units do not have any control task. Master units can be PLC, computer or visual control units. The objective of the master unit is to provide the control. With the help of slave stations, the system can be gathered at one point and from this point the whole system can be observed. In Figure 12 a master-slave structure is showed. In this master-slave structure, there is a necessity to use a protocol for the intercommunication. By this communication, all wires will be eliminated and control will be maintained with only a cable. With this communication also the information security will be increased.

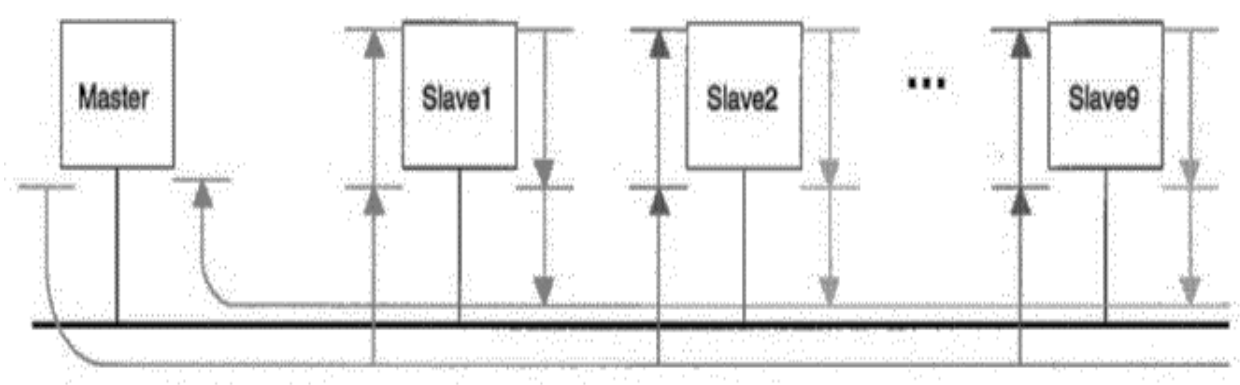

Fig. 12. Master-slave structure (F.L. Lian, J. Moyne, and D. Tilbury, 2002)

There is a hierarchical order in a distributed control system and this system's function units are placed distributed. In these infrastructures, there are powerful and autonomous automation systems. Domain points or control loops $(1,8,16,32)$ are restricted by the measuring point. The infrastructures, which are functionally autonomous and placed in this area, are called field stations. The first task of the field station is:

- To do the preliminary check by collecting analog and digital signals.

- Transmission of the tracking and the field messages.

- To do the open and closed-loop control processes.

Every server has two backed up a server in the condition of not exceeding one or two keys. At the same time, all control systems are connected to the link server. These keys help to the communication between the client and the controller. Distributed control systems can operate one or more than one workstations and can be configured by a personal computer at the workplace. Extra computers, which have the abilities of data collecting and reporting, can be added to a server and/or applications. In typical distributed control systems, there are regulatory control cycles that have the executive ability between 1-256 and distributed to a geographical area (M. Anand, S. Sarkar, and S. Rajendra, 2012).

\section{IMPLEMENTED SYSTEM DESIGN}

\subsection{Factory System Design}

The main structure of the designed system is shown in Figure 13. If we set forth from the picture, the places where people work, numbered 2 and 3, are called stations. At the beginning of these stations, there are conveyor belts carrying products. At the stations generally, the packaging process is done by operators and the products stands in front of them are put into their places. There is a forklift to carry the products to the logistics department in the factory. In our designed system there are three stations. At every station, there are laser distance sensors thought about to check products' fullness ratio. In the system, there is a wireless sensor network structure, and fullness ratio information is transmitted via wireless nodes. At the same time, to check the forklifts if they are coming to the right station, there are RFID read/write tags exist on forklifts. Automatic and manual working modes are aforethought together in the system. Manual mode is developed to intervene in case of a breakdown. In automatic mode, the system works by itself until the factory is entirely stopped. When the factory is opened, operators set the system to automatic mod and system stays active for the whole day.

\subsection{Sample Code Algorithm}

For the designed system first of all a control algorithm illustrated in Figure 14 is designed. This control algorithm will be explained part by part. The first control algorithm is created to decide according to the fullness ratio of the products at the stations. At every station, there are laser distance sensors to observe the fullness ratio. From the operator panel, according to daily working conditions, a limit fullness ratio will be entered. For example, on the $1^{\text {st }}$ day, if a quick product shipment is wanted, the limit of fullness ratio should be entered lower. If there is no such priority, a normal limit value may be entered. It must be decided only according to working conditions. Another possibility is that at every station there are different products are produced. In such a case, some 
products may be maybe desirable in more copious amounts depending on days. The limit value entered from the operator panel can solve this problem too. All algorithms are created for one station, and the control of all stations are same.

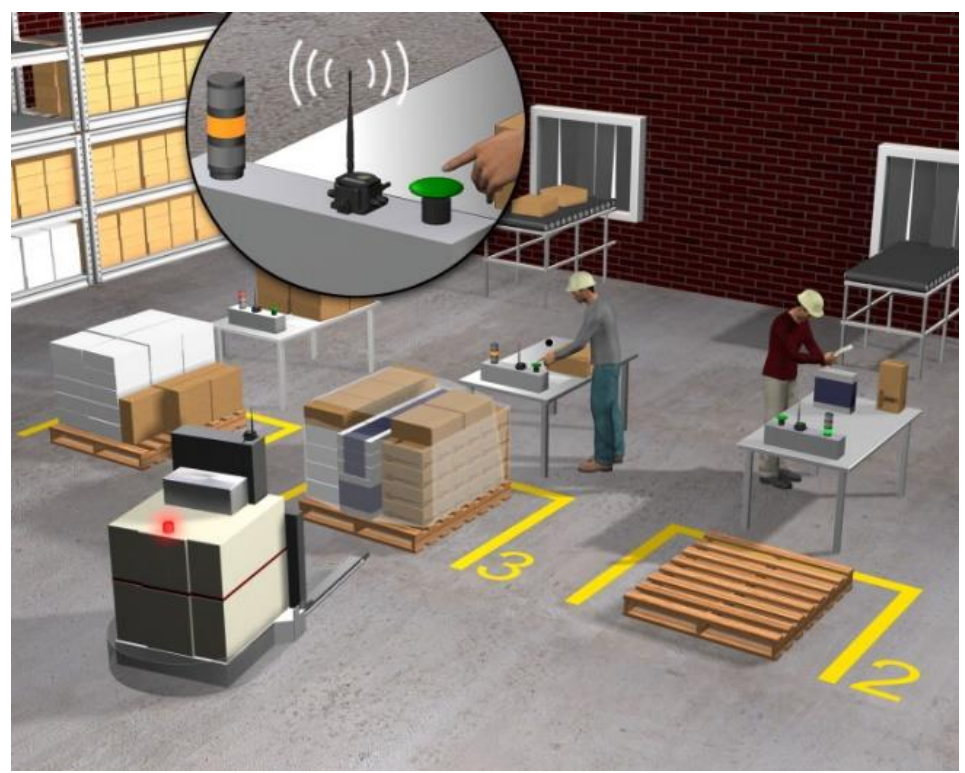

Fig.13. Sample factory environment (M. Raffin, 2016)

Forklifts perform the necessary tasks by steering to the station where the information comes. However in case of a mishap or when the operator perceives the following information wrong they can be misdirected. To prevent these, some other technologies can be applied. The most up-to-date method for such applications is RFID technology. This technology can read and write with radio frequency and has its unique tags. In these tags, there are specific memories. For example, if a name wanted to be written in it, its memory should be more significant. However, if only a number wanted, a regular size of memory can be chosen. There are reading/writing nobs to do the reading and writing processes. These are divided into two according to their detection distance such as HF (high frequency) and UHF (ultra high frequency). E.g., at the entrances of highways, there are UFH technology is being used. In order to adapt to our application, tags are attached to every station, and there are two reading/writing nobs on forklifts. At the installation phase, a code is given to every tag at stations, and they are stored. In the PLC program when a forklift goes to the wrong station, operators must be warned. This program will be explained more detailed at the PLC part. A sample RFID algorithm is shown in Figure 15.

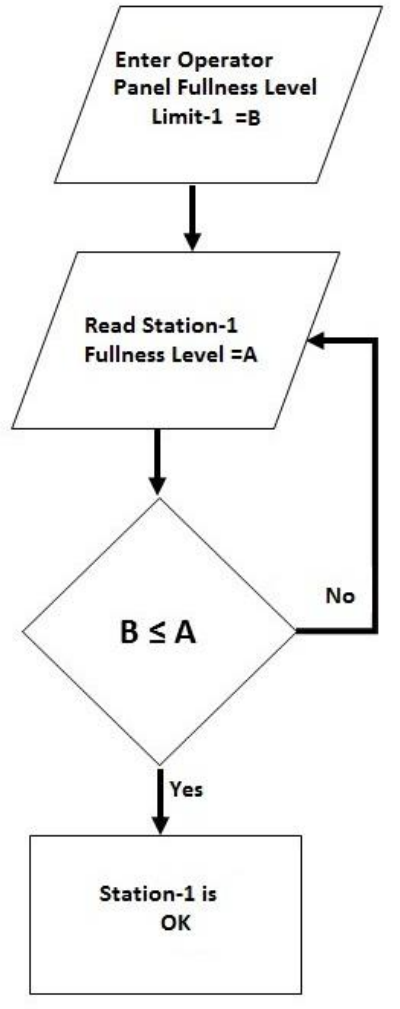

Fig. 14. Fullness control of station

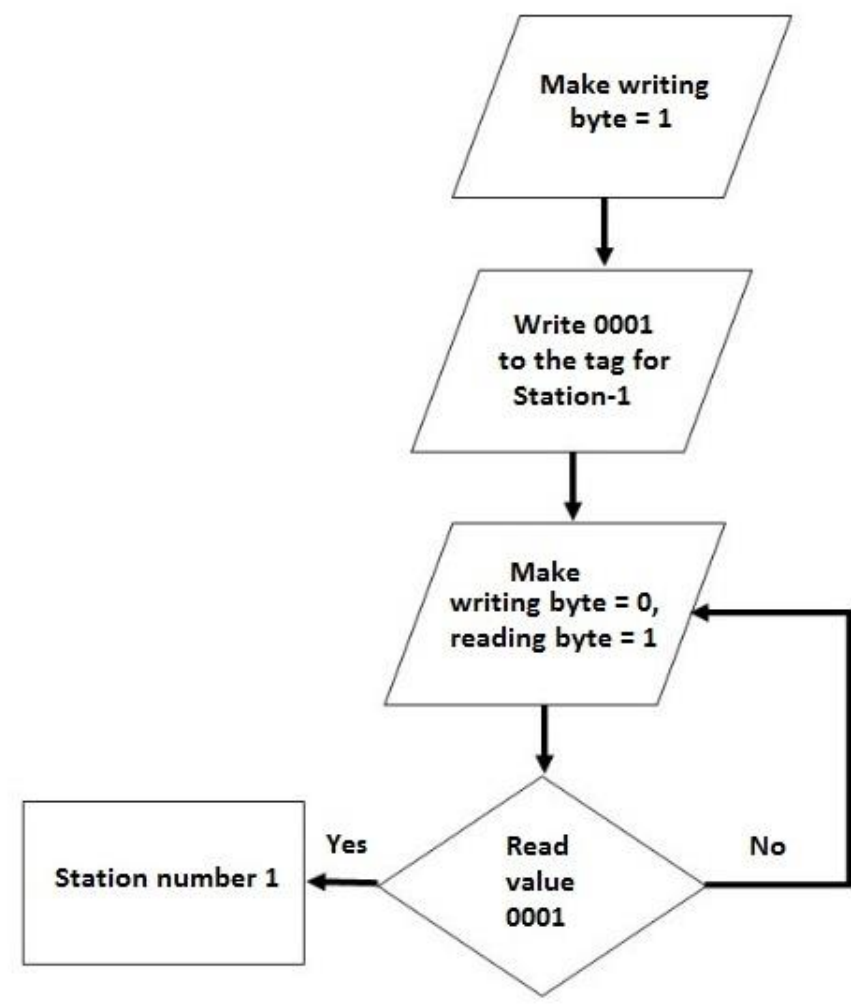

Fig. 15. Writing the PLC Program with Codesys 


\subsection{Writing the PLC Program with Codesys}

Analog 4-20 mA signal corresponds to the numerical value 0-32767 in the PLC program. This value's type is the word in PLC. Firstly it should be converted into REAL type. Expressing the values in decimal value is critical. After that, by using simple mathematical operations, this value is converted. As can be seen in Figure 16, the value 'istaston_1_lazer_sensör' is coming 20000 from the field, and when this value put on the formula the real measuring value is found $610 \mathrm{~mm}$. This value, which is in REAL data type, can be used in every POU. The use of RFID technology is mentioned in the algorithm part. Detailing the subject, in RFID tags there are memories to write some values. By activating specific letter can be written how much of these memories can be used. These bytes are called 'byte_count,' and there are 3 of them. If ' 1 ' is written in these 3 bytes, all of the memory becomes available. In RFID structure, choosing reading or writing processes is related to the bytes to be activated. There are two bytes for reading and writing. Firstly writing byte is activated and codes are written in the tags. After writing the codes, with the codes read in the program, and at the stations, true-false control is made. In Figure 17 at the section number one, the activation of writing byte is seen. When the reading byte is activated in the same way, the writing byte is deactivated automatically. In section 2, significant bytes to activate are shown to use the memory part.

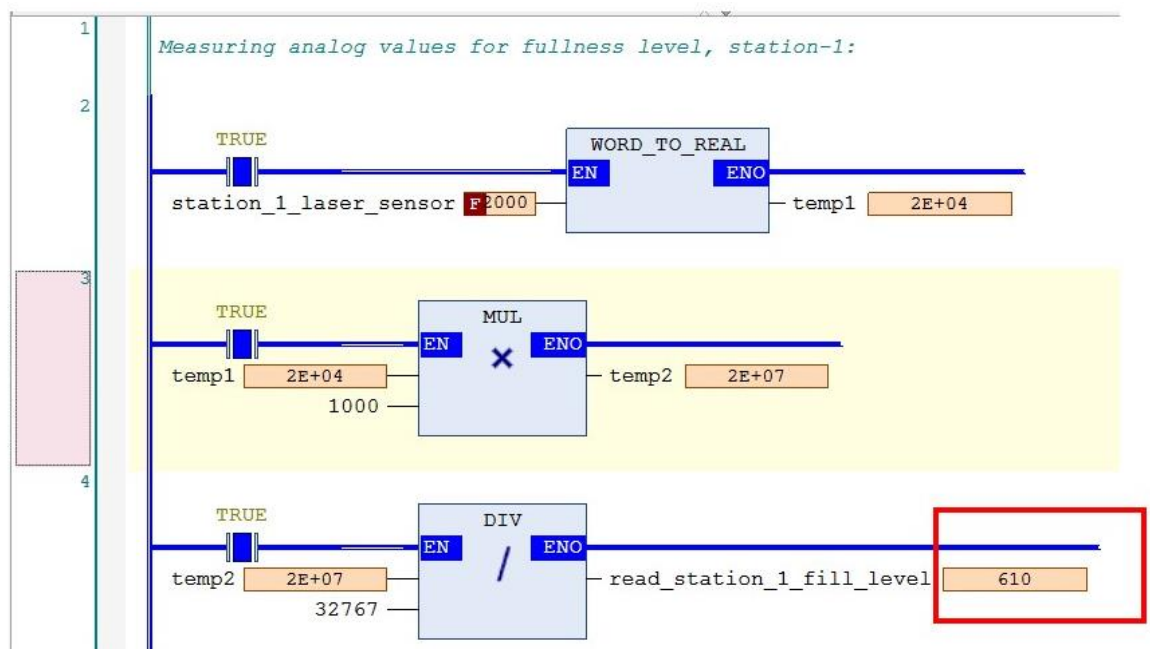

Fig. 16. Analog signal processing

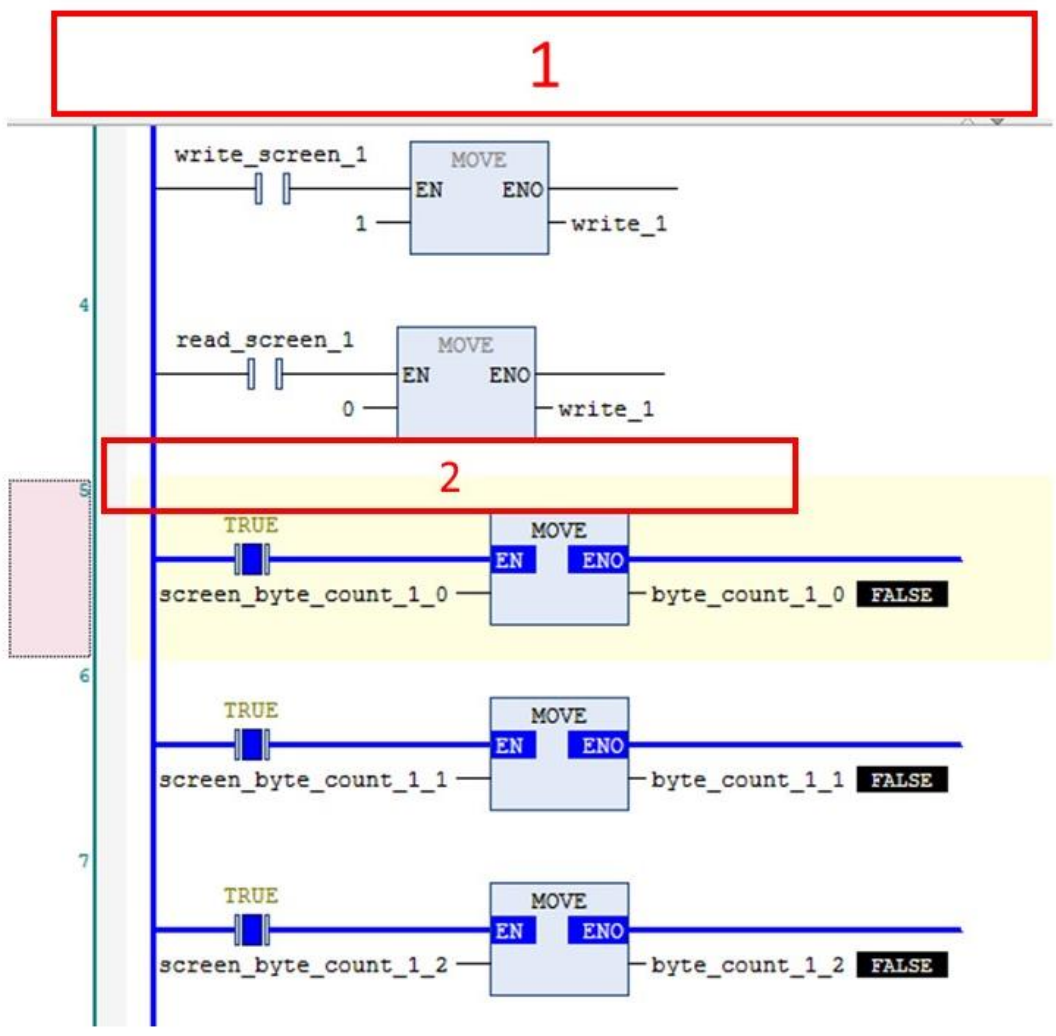

Fig.17. RFID project-1 
At the third section in Figure 18, activation of the reading/writing nob with the help of the sensing unit exists in the forklift, is illustrated. At the fourth section, a value is written for the code to be given to the particular station. At the fifth section, it is shown how to read from the tags codes written in it. At the sixth section, a comparison made between the read and present values and a result about stations truth is obtained.

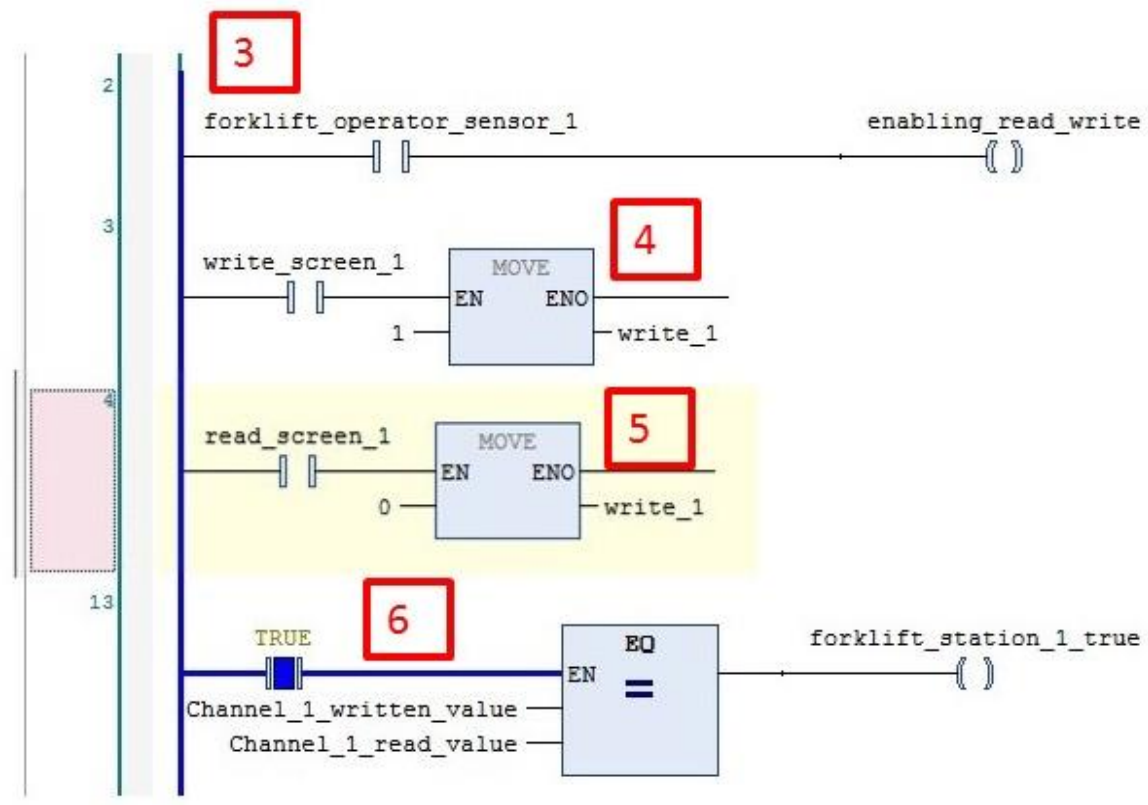

Fig.18. RFID project-2

\subsection{Control of the System with Fuzzy Logic}

In a factory system, forklifts carry the products with manual control logic. To make it clear, at two of the stations when the products are ready, forklift operators are going to the station the first one they see or without any priority. This manual control logic causes inefficiency and energy loss at systems. In order to prevent this situation, a fuzzy logic approach is tried for this manual control. A decision-making mechanism algorithm is developed for the forklifts to choose a station which one to go and take the product. When the movements of the forklifts are considered, taking the products from the stations without any delays is a critical expectation. To meet this expectation, parameters about the forklift movement, which creates the fuzzy logic model, become very important. In Figure 19, inputs and outputs of the fuzzy logic are illustrated.

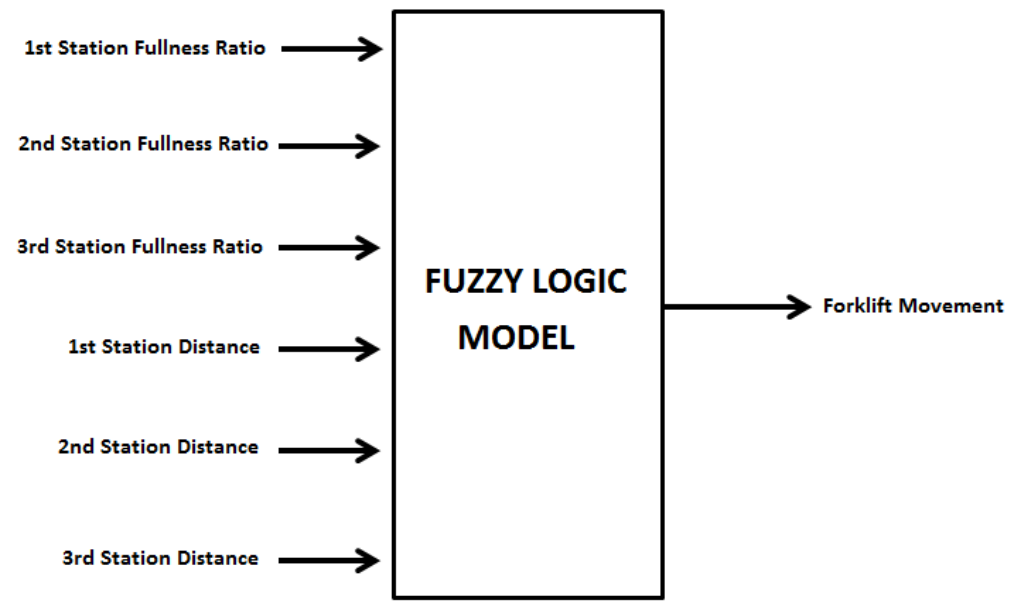

Fig. 19. Input and output parameters of the system

The most successful directing with fuzzy logic model belongs to the forklift movement, according to finishing ratio of the products and the distance between forklifts and the station, is aimed. A laser distance sensor is used in order to determine the parameters in the system. By using the output of the sensor, the parameters are obtained during the movement of the forklift. As an input to the system, determination of the fullness ratio at the stations is given. As another input, 6 inputs are determined by obtaining the distance between the forklifts and the stations. With this information, movement of the forklift is decided. Forklift's movement is assigned as one input in the system.

While designing the system and creating the fuzzy logic block diagram, a suitable software program is used. The fuzzy logic control, which its rules were created, is evaluated by two different methods. These methods are called Mamdani and Sugeno. 
Mamdani method needs supervisor information, it is commonly used and can be used at every problem-solving. Sugeno is used in the applications with fewer variables. At the same time, fuzzification and defuzzification processes can be done in the software program. In Figure 20, a screenshot of the designed page is given. As it can be seen on the editor's page six inputs and one output is defined. Besides, Mamdani method is used and named as "Forklift application 1". The next step is creating membership functions in input and output functions. The software editor, which is used, lets us choose the type of the function. The most used type is a triangle type, and this type is used in this application.

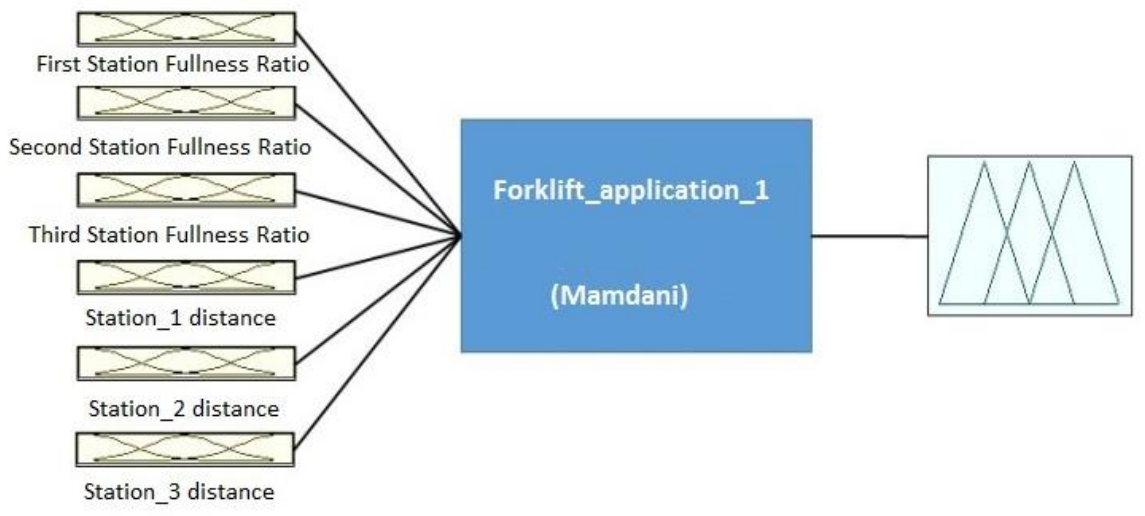

Fig.20. Fuzzy logic editor

For the fullness ratio of the stations defined as an input, three membership functions are assigned. Membership functions are divided into three parts such as low, medium, high. This input value is given in percentage. Membership functions of this input are given in Figure 21. These membership functions are the same for these three stations.

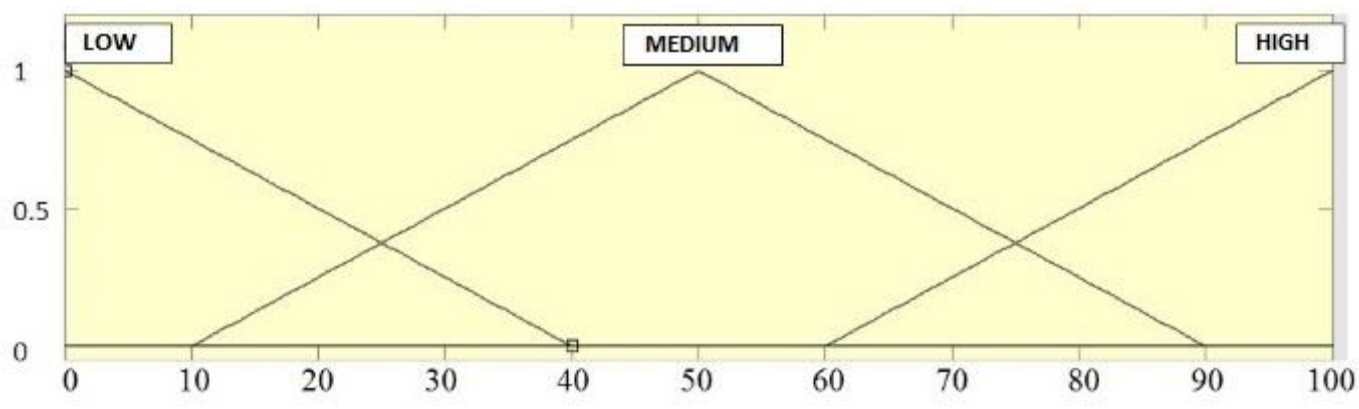

Fig.21. Membership functions belonging to station fullness ratio

Three membership functions are defined for the forklift's movement, defined as the output. Membership functions are divided into three parts as forklift's movement station1, station2, and station3. These output values are defined between 0 and 3. Membership functions of the output variable are given in Figure 22.

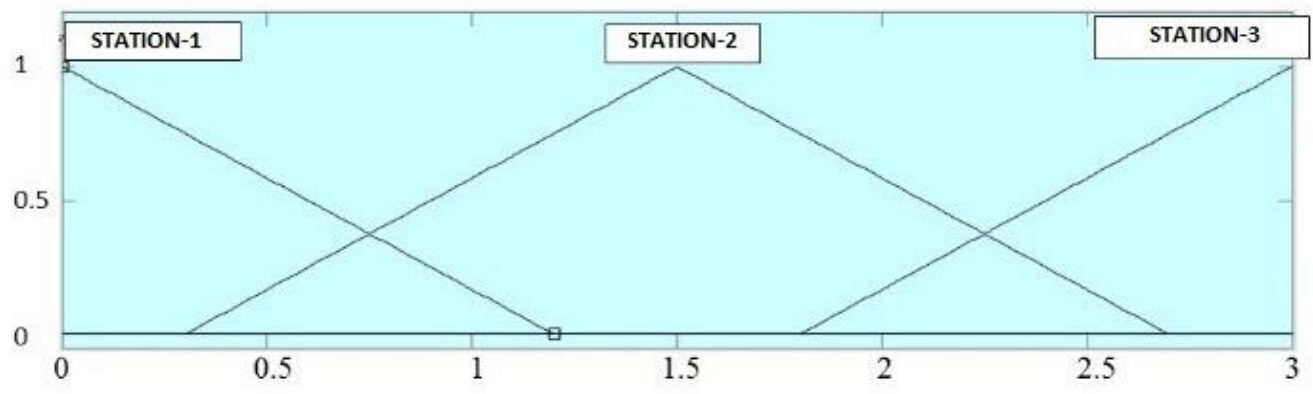

Fig.22. Membership functions belonging to forklift's movement

In order to create the model, after creating the membership function, 12 rules are created to determine the necessary interactions between parameters. These rules are created according to the supervisor's information, and the scenarios can happen in the field are considered. For example, these rules can be listed like; 
- If the fullness ratio of the first station is high and the fullness ratio of the second station is low, and the fullness ratio of the third station is high, and the distance to the first station is short and the distance to the second station is far, and the distance to the third station is far, forklift go to the first station.

- If the fullness ratio of the first station is low and the fullness ratio of the second station is high, and the fullness ratio of the third station is low, and the distance to the first station is far and the distance to the second station is short and the distance to the third station is far; forklift go to the second station.

- If the fullness ratio of the first station is low and the fullness ratio of the second station is low, and the fullness ratio of the third station is high, and the distance to the first station is far and the distance to the second station is far and the distance to the third station is short; forklift go to the third station.

- If the fullness ratio of the first station is high and the fullness ratio of the second station is high, and the fullness ratio of the third station is medium, and the distance to the first station is medium and the distance to the second station is medium and the distance to the third station is medium; forklift go to the first station.

- If the fullness ratio of the first station is high and the fullness ratio of the second station is high, and the fullness ratio of the third station is low, and the distance to the first station is short and the distance to the second station is far and the distance to the third station is medium; forklift go to the first station.

As a result of these fuzzy rules, rule visualizer is shown in Figure 23. Rule visualizer includes the entire program until the rules are made. The part number one, so the yellow graphs in the first six columns of the figure show the membership functions belong to the rule entries. Blue graphs in the seventh column show the membership functions dependent on the result values of output. The blue graph right below the third part shows how every output became integrated and defuzzification. When pressed on the entries by making some changes on the entries, a change on integrated output value (the red line) is observed. In this way, rule visualizer gives the opportunity of explication about the changes and fuzzy systems. The information received from the rule visualizer is explicated according to the information below.

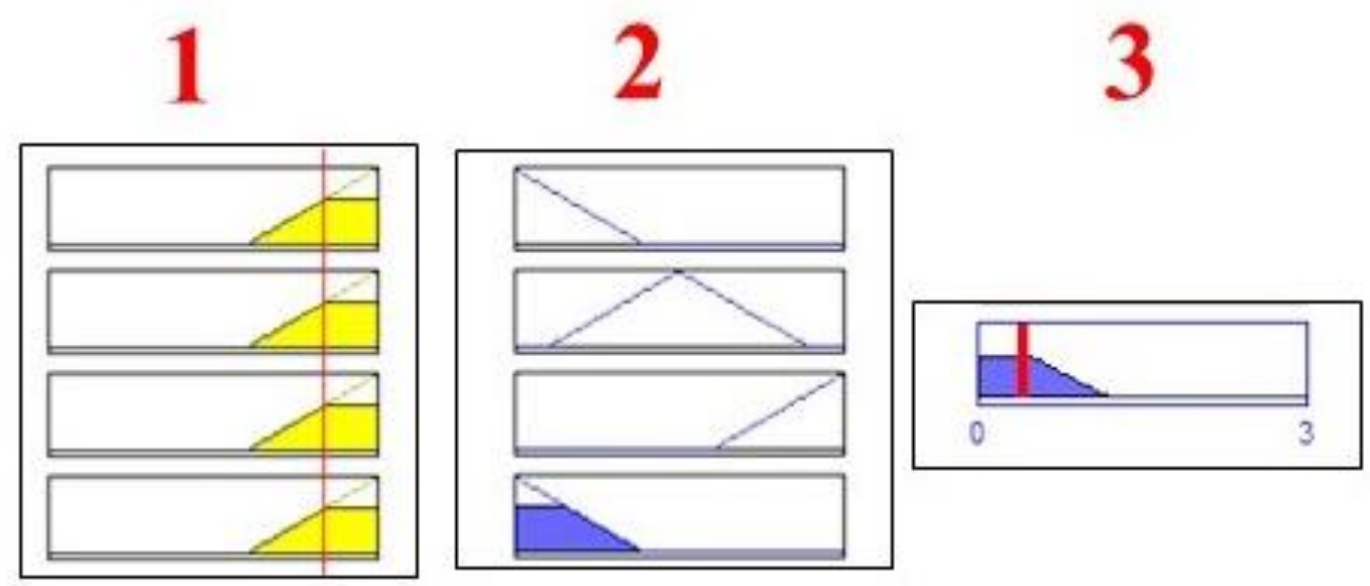

Fig.23. Fuzzy system rule visualization screen

Rule visualizer calculates the single result of the fuzzy inference system. If to see the surface, consists of the values of the output taken from the fuzzy inference system are required, surface visualizer is needed. The solution surface, in the fuzzy system solution of the problem considered in this part, is visualized. The surface at the graph helps to interpret the outputs, which of them are how related with which of the inputs and which of them are effective or ineffective within which values. If the surface visualizer in Figure 24 is examined, it can be observed that when the first and the second stations' fullness ratios are low, forklift goes to the third station.

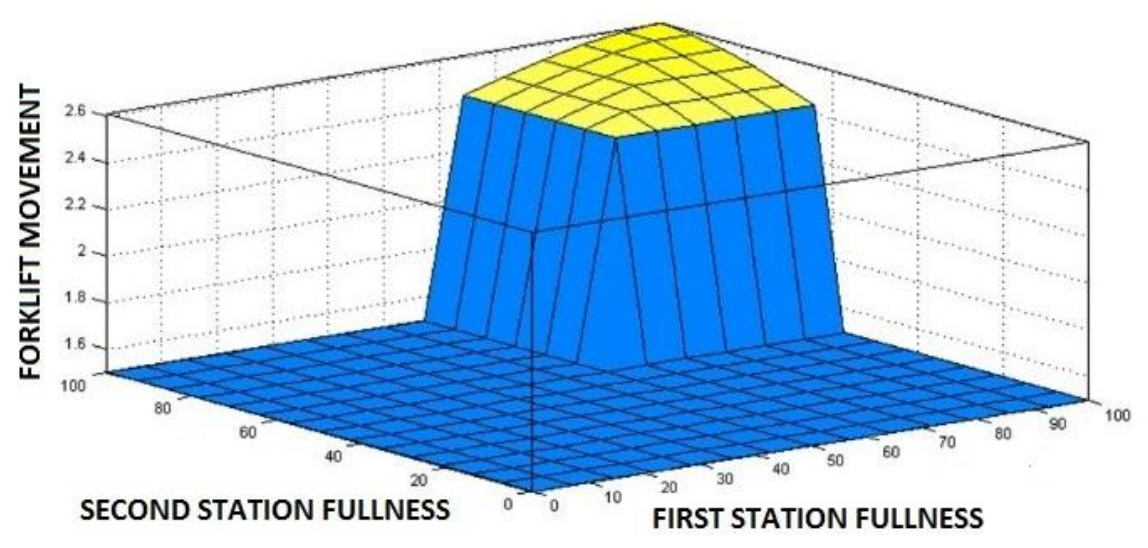

Fig.24. Surface visualiser-1 
At the surface visualizer in Figure 25, shows that the fullness ratio of the first station is almost whole, distance to the second station is between short and medium. At this situation, the forklift is going towards to the first station.

In Table 1, output values, created by using the rules and the membership functions defined for the input and output parameters corresponding to the values given to the fuzzy logic model, is given. For example, at this table, if the $6^{\text {th }}$ row is examined, although the fullness ratios are almost similar, the fuzzy model directed the forklift to the second station because the distance is closer. Again in the same way if the values at the $8^{\text {th }}$ row are interpreted, the fullness ratio of the third station is four times bigger. When we look at the distances from the location of the forklift, it is close to the first and the second stations, and it is in the middle. If there was not fuzzy logic, the operator could have chosen the closer stations, but when we examine the products, the products at the third station is two times more than the sum of the products at the first and the second stations. From here it can be observed that how successful, and the fast result is given by the model.

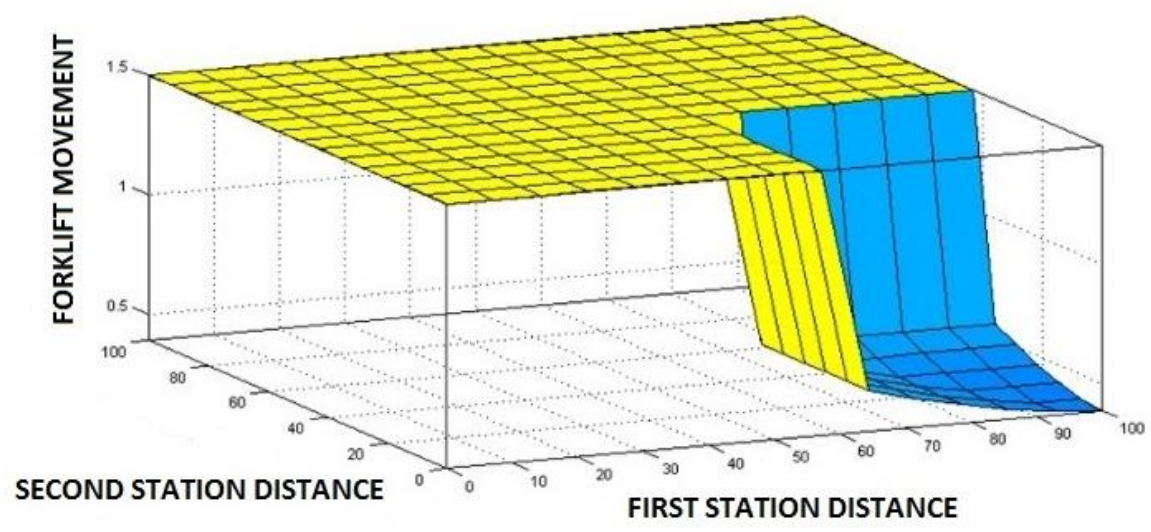

Fig.25. Surface visualizer-2

\subsection{The Performed System Simulation}

At the simulation, when the products at the stations are finished. This information is sent to the free forklift. The free forklift gets this information and the sensor attached to the forklift automatically deletes the information going to the forklift. In Figure 26, a software program created to make stations ready is seen in a simulation environment. For the fullness level, 610 value is being received from the laser distance sensor. From the screen, the limit is set to 600. As a result the output of the first station is activated.

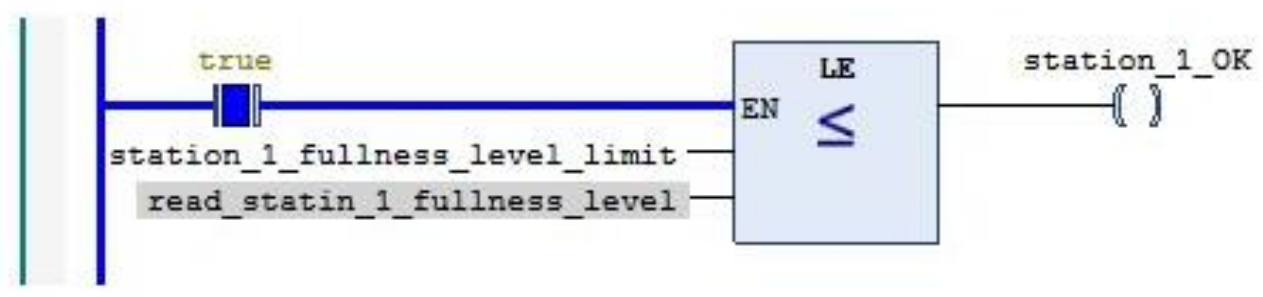

Fig. 26. Making stations ready

When the output of the first station is activated if the automatic conditions are met, the first step of automatic movement is activated. Simulation of the software is given in Figure 27.

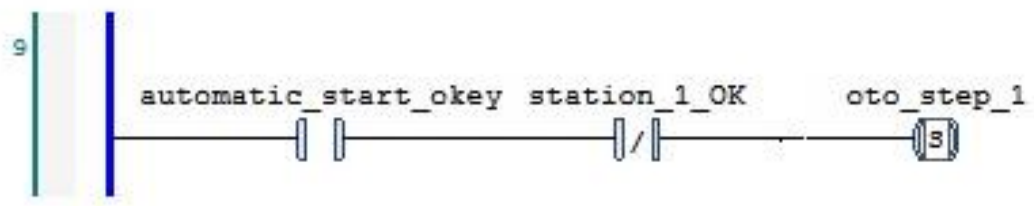

Fig.27. The first step of the automatic movement 
Table 1. Results are given by the fuzzy logic model corresponding to the given input and output values

\begin{tabular}{|c|c|c|c|c|c|c|c|}
\hline \multicolumn{6}{|c|}{ Inputs } & \multirow{2}{*}{\begin{tabular}{|c|} 
Outputs \\
\\
Forklift \\
Movement
\end{tabular}} & \\
\hline $\begin{array}{c}\text { Completion } \\
\text { of the } \\
\text { first station }\end{array}$ & $\begin{array}{c}\text { Completion } \\
\text { of the } \\
\text { second station }\end{array}$ & $\begin{array}{l}\text { Completion } \\
\text { of the } \\
\text { third station }\end{array}$ & $\begin{array}{l}\text { Distance the } \\
\text { first station }\end{array}$ & $\begin{array}{c}\text { Distance the } \\
\text { second station }\end{array}$ & $\begin{array}{l}\text { Distance the } \\
\text { third station }\end{array}$ & & \\
\hline $78,90 \%$ & $13,40 \%$ & $15,90 \%$ & 14,3 & 87,4 & 83,9 & 0,465 & 1.station \\
\hline $25,40 \%$ & $72,80 \%$ & $15,90 \%$ & 83,2 & 15,1 & 83,9 & 1,5 & 2.station \\
\hline $35,80 \%$ & $35,80 \%$ & $85,80 \%$ & 85,8 & 78 & 34,9 & 2,44 & 3.station \\
\hline $84,10 \%$ & $83,20 \%$ & $22 \%$ & 18,5 & 86,6 & 76,3 & 0,923 & 1.station \\
\hline $22 \%$ & $83,20 \%$ & $85,80 \%$ & 88,4 & 11,6 & 93,5 & 1,5 & 2.station \\
\hline $68,50 \%$ & $73,70 \%$ & $72,80 \%$ & 38,4 & 34,2 & 39,2 & 1,63 & 2.station \\
\hline $94,40 \%$ & $79,70 \%$ & $61,60 \%$ & 26,3 & 44,4 & 40,9 & 2,52 & 3.station \\
\hline $29,70 \%$ & $21,10 \%$ & $88,40 \%$ & 26,3 & 22 & 63,4 & 2,07 & 3.station \\
\hline $18,50 \%$ & $64,20 \%$ & $66,80 \%$ & 30,6 & 22 & 62,5 & 2 & 2.station \\
\hline $67,70 \%$ & $39,20 \%$ & $22 \%$ & 32,3 & 22 & 62,5 & 0,537 & 1.station \\
\hline
\end{tabular}

Due to the second step has no wireless connection with the conveyor movement the step is skipped to the third step. The third step activates the information sending output to the free forklift. In Figure 28, activation of the information sending output is shown.

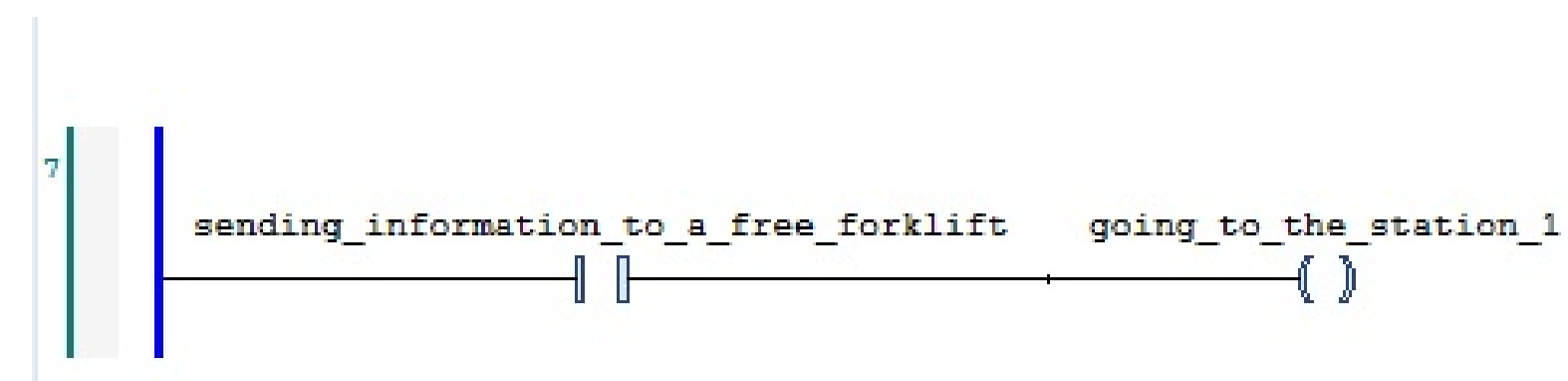

Fig. 28. Activation of the wireless sensor output

Finally, when the information sending output is activated, wireless sensor output sends the command of 'go to the station 1' to the free forklift (Fig. 28.). This simulation does both movements directing and wireless sensor network structure test. As a result, it is observed that the correct results are obtained at the simulation. By considering every possibility can occur in the system, the software was run and directing is done.

\subsection{Obtained Results}

System optimization is achieved by applying fuzzy logic control for the forklift's movement. At the end of this optimization, some deductions were made. Profit graphs are plotted with the obtained results. Comparison of fuzzy and classical logic is made, and the obtained results are shared (Fig. 29.). At the graph forklifts' daily directions to the stations are shown. The profits between classical logic and fuzzy logic are shown up as a percentage. For the forklift's movement, fuzzy logic has been a good solution. 


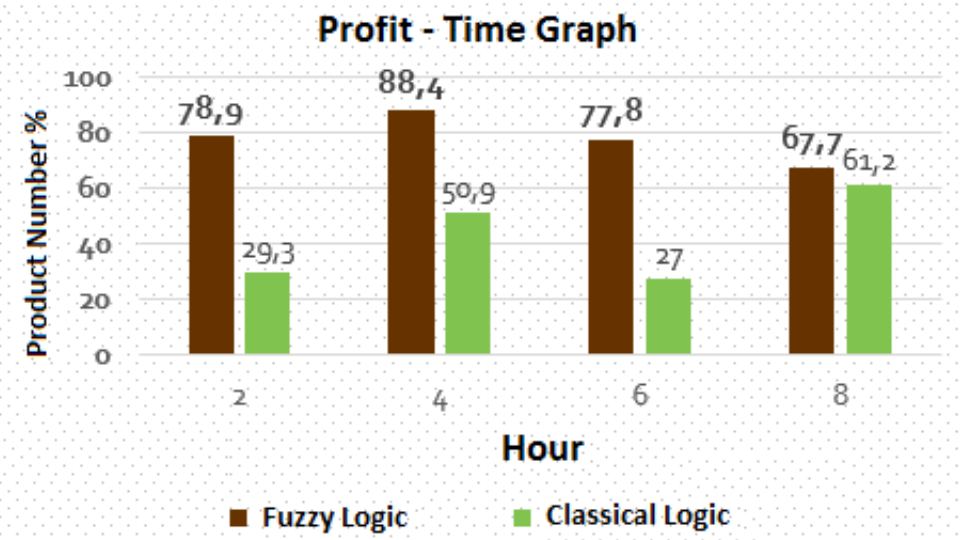

Fig. 29. The profit graph

In Figure 30 weekly profit graph is given. Weekly high levels of profits are obtained with fuzzy logic.

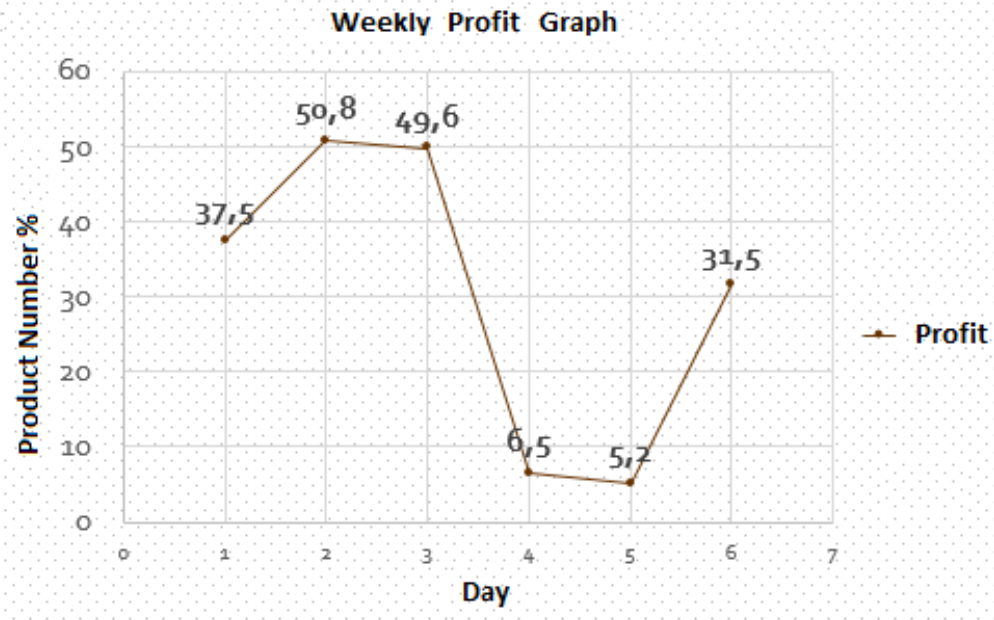

Fig.30. Weekly profit graph

In Figure 31 monthly profit graph is given. At this graph, there are immediate changes. The reason for these changes is there are profits in some situation, but it has a lower percentage. This caused ups and downs at the graph.

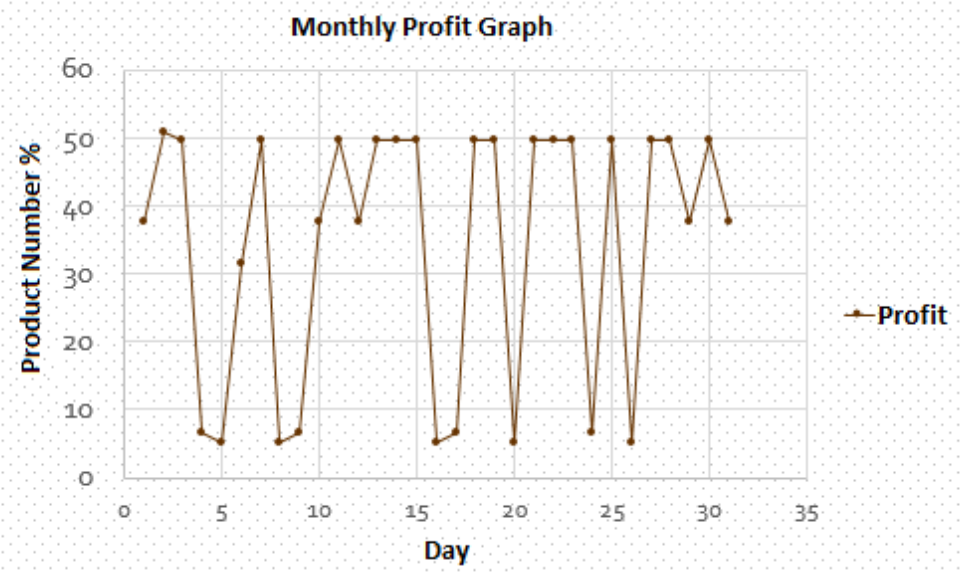

Fig. 31. Monthly profit graph

The graphs given until here give profits as materials. At the same time in some cases, forklift does twice expedition. Fuzzy logic decreases this number to 1. In Figure 32 profit graph regarding cost is given. 


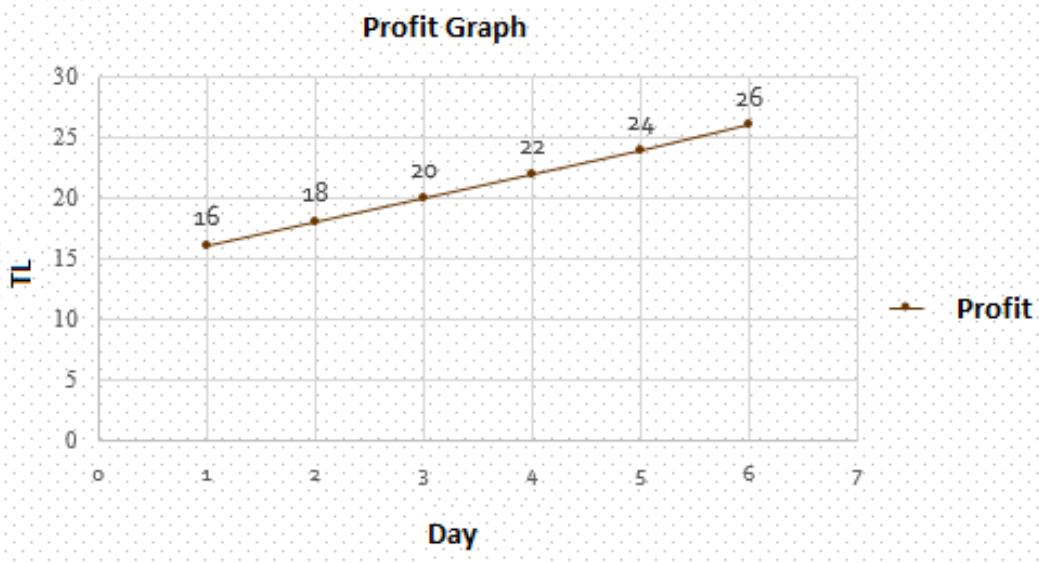

Fig. 32. Cost - profit graph

\section{RESULTS AND SUGGESTIONS}

In this study, a factory designed and by applying different control methods to this design, some results are obtained. First of all the algorithmic structure is determined and created. With this algorithmic structure, many different control systems can be created. The important fact is to choose the most suitable method according to the operation and increase the efficiency to the highest rates. The control done by PLC program gives successful results in many fields. At the algorithms, which a digital signal relation is set up, it gives very successful and secure results. With the help of this software, users have a wide range of uses and have an easiness to change the system. Besides, PLCs and operator panels have more possibilities to work together. The fact operator panels can quickly adapt to PLCs and their abilities to work together make a significant contribution to systems. The software can be written in the Codesys program gives the easiness to create the system. With this program, a program for automatic movement in a factory is written, and a successful result is obtained. However, a random a classical digital logic is created according to a random emptyfull logic for the movement of the forklift. This causes some problems at increasing efficiency. A fuzzy model is designed in order to eliminate these problems. Via this model, according to the forklifts distances from the stations and the products fullness ratios at the stations, an economical forklift movement is aimed. When it is necessary, additions and subtractions can be done to the parameters such as distances of the forklifts determined as input and the product-finishing ratio of the stations, and the forklift movement determined as output. Energy and time saving is aimed by delivering the products faster without any delays with the control of the output parameter. By creating an optimum wireless sensor network and sending the sensor information to the forklift faster and in this way widening the forklift's movement area is aimed. Thus without any human intervention to the parameters, the forklift movement can be done by automatically setting with the machine.

As a result, it is observed that the control with fuzzy logic gives better results than controlling with PLC and classical logic control. Besides, in the factories (or the places where process automation is widely used), the fact that fuzzy logic control can be applied easily is seen. It has been a different and innovative approach to the forklift movement.

\subsection{Suggestions}

It is possible to increase the optimization for the fuzzy logic approach to forklift movement by adding more input parameter like finishing time of the products. Besides the supervisor information about the system can be widened and membership functions of the inputs can be increased so that it will increase the control precision. With the outputs obtained from the fuzzy inference, a specific function structure can be created and according to this function PLC software can be created, in this way control will be removed from the classical logic. By increasing the supervisor information, the rule base can be increased more, and on this even, the most exquisite detail will be organized according to this control. Forklift movement can be made autonomous, and the delays caused by operators can be minimized. According to the conditions of the application, the type of communication of wireless sensors can be chosen differently. A different suggestion, a function can be created by the outputs obtained via fuzzy logic and can be written in a PLC software, because with the help of the defuzzification stage, outputs can be obtained as numerical values.

\section{REFERENCES}

Anand M., Sarkar S., and Rajendra S., Application of Distributed Control System in automation of Process Industries, International Journal of Emerging Technology and Advanced Engineering 2 (2012), 377-383.

Arınç R., Bulanık mantık yöntemiyle asenkron motor hız kontrolü ve simülasyonu, Master Thesis, Marmara University, 2003.

Bai Y., Zhuang H.Q., and Roth Z.S., Fuzzy logic control to suppress noises and coupling effects in a laser tracking system, Ieee Transactions on Control Systems Technology 13 (2005), 113-121. 
Bolton W., Programmable logic controllers, in Programmable Logic Controllers (Fourth Edition), Newnes, Oxford, 2006 , pp. 1 16.

Chanak P. and Banerjee I., Fuzzy rule-based faulty node classification and management scheme for large-scale wireless sensor networks, Expert Systems with Applications 45 (2016), 307-321.

Choi G.H., Choi G.S., and Jang J.H., A Framework for Wireless Sensor Network in Web-based Monitoring and Control of Indoor Air Quality (IAQ) in Subway Stations, 2nd IEEE International Conference on Computer Science and Information Technology (ICCSIT) (2009), 378-382.

Demirel F., Su İletim Hatları Basınç Bilgilerinin Deneysel Bir Ortamda Kablosuz Sensör Ağ ile Toplanması, Master Thesis, Ege University, 2010.

Dumitru I., Arghira N., Fagarasan I., and Iliescu S., A fuzzy PLC control system for a servomechanism, IFAC Proceedings Volumes 43 (2010), 69-74.

Faludi R., Building Wireless Sensor Networks, O’Reilly Media USA, 2010.

Kılıç A.B., RFID Yoklama Otomasyonu, Master Thesis, Kırıkkale University, 2015.

Kurtulan S., PLC ile Endüstriyel Otomasyon, İstanbul, Birsen Yayınevi, 2010.

Kuvulmaz J., Endüstriyel Prosesler İçin PID Tipi Bulanık Kontrolör Tasarım, Yüksek Lisans, Marmara Üniversitesi, 2002.

Labrador M.A. and Wightman P.M., Topology Control in Wireless Sensor Networks, Springer Netherlands, 2009.

Lee C.K.H., Choy K.L., Law K.M.Y., and Ho G.T.S., Application of intelligent data management in resource allocation for effective operation of manufacturing systems, Journal of Manufacturing Systems 33 (2014), 412-422.

Lian F.L., Moyne J., and Tilbury D., Network design consideration for distributed control systems, Ieee Transactions on Control Systems Technology 10 (2002), 297-307.

Lin J. and Lian R.-J., Self-organizing fuzzy controller for injection molding machines, Journal of Process Control 20 (2010), 585595.

Mainwaring A., Polastre J., Szewczyk R., Culler D., and Anderson J., Wireless Sensor Networks for Habitat Monitoring, WSNA’02 (2002).

Morsi I. and El-Din L.M., SCADA system for oil refinery control, Measurement 47 (2014), 5-13.

Ozbek G., Radio Frequency Identification (RFID) Systems Implementation on Defence Industry, Master Thesis, Selçuk University, 2014.

Özek A. and Sinecen M., Klima Sistem Kontrolünün Bulanık Mantık ile Modellenmesi, Mühendislik Bilimleri Dergisi 10 (2004), 353-358.

Raffin M., Wireless Webinar, in, 2010.

Raffin M., Shopping Center Directs Visitors to Available Parking Using Banner Wireless, in, USA, 2016.

Siddique N., Intelligent Control, Springer, Poland, 2013.

Singhala P., Shah D., and Patel B., Temperature Control using Fuzzy Logic, International Journal of Instrumentation and Control Systems 4 (2014), 1-10.

Soylu T., Kablosuz Algılayıcı Ağların Uygulama Alanları ve Bir Algılayıcı Düğüm Tasarımı, Master Thesis, Trakya University, 2012.

Tiryaki E.A. and Kazan R., Bulaşık Makinesinin Bulanık Mantık ile Modellenmesi, Mühendislik ve Makine 48 (2007), $3-8$.

Üstündağ A., Radyo Frekans Tanıma (RFID) Teknolojisinin Tedarik Zinciri Üzerindeki Etkileri, PhD Dissertation, İstanbul Technical University, 2008.

Yazar M., Kablosuz Sensör Ağları ile Yangın Uyarı Sistemi, Master Thesis, Yıldız Technical Univesity, 2015.

Yick J., Mukherjee B., and Ghosal D., Wireless sensor network survey, Computer Networks 52 (2008), $2292-2330$. 
Yılmaz S., Bulanık Mantık ve Mühendislik Uygulamaları, KOÜ Yayınları, 2007. 